\title{
Pharmacological Interventions for Treatment of Sarcopenia: Current Status of Drug Development for Sarcopenia
}

\author{
Ju Yeon Kwak ${ }^{1}$, Ki-Sun Kwon ${ }^{1,2}$ \\ ${ }^{1}$ Aging Research Center, Korea Research Institute of Bioscience and Biotechnology, Daejeon, Korea \\ ${ }^{2}$ Department of Functional Genomics, KRIBB School of Bioscience, University of Science and Technology, Daejeon, Korea
}

Corresponding Author:

Ki-Sun Kwon, PhD

Aging Research Center, Korea Research

Institute of Bioscience and

Biotechnology, 125 Gwahak-ro,

Yuseong-gu, Daejeon 34141, Korea

E-mail:kwonks@kribb.re.kr

ORCID:

https://orcid.org/0000-0002-6401-2735

Received: July 17, 2019

Revised: August 7, 2019

Accepted: August 10, 2019

\begin{abstract}
Sarcopenia, the loss of skeletal muscle mass and function with age, was first recognized as a disease in the International Classification of Diseases, Tenth Revision, Clinical Modification (ICD-10CM) (M62.84) and has recently attracted attention as aged populations increase. However, the diagnostic criteria for sarcopenia remain controversial and there are as yet no US Food and Drug Administration-approved medications for sarcopenia. Given that both intrinsic and extrinsic factors contribute to sarcopenia onset and development, understanding the mechanism of sarcopenia is important for the development of therapeutic strategies. In this review, we described a variety of drugs for sarcopenia under investigation, including myostatin/ActR2 signaling inhibitors, exercise mimetics, anabolic hormones, and natural compounds. However, the combination of non-drug therapies with exercise and nutritional supplements are also needed as more easily accessible intervention strategies against sarcopenia rather than pharmacological treatments alone. Many approaches to develop therapeutic methods to overcome sarcopenia may lead to healthy aging.
\end{abstract}

Key Words: Sarcopenia, Skeletal muscle, Aging, Diagnosis, Drug

\section{INTRODUCTION}

Sarcopenia is the decline in skeletal muscle mass and function with age. ${ }^{1)}$ Muscle mass and strength peak in early adulthood, followed by a gradual decline after 40 years of age, ${ }^{2)}$ with a more substantial decline from the fifth decade onwards. ${ }^{3)}$ Sarcopenia has been considered a consequence of normal aging; however, research on sarcopenia has supported its recognition as a disease entity, with a new disease code in the International Classification of Diseases, Tenth Revision, Clinical Modification (ICD-10-CM) (M62.84) established in October 2016.

Currently, the suggested clinical diagnostic criteria for sarcopenia are based on low muscle mass and function (strength or performance $)^{5)}$ although an international consensus is needed regarding the specific criteria and cut-offs. The technologies used to estimate muscle mass include magnetic resonance imaging, computed tomography, dual-energy X-ray absorptiometry scans, and bioelectrical impedance analysis. The parameters to test muscle strength and performance are handgrip strength and gait speed, respectively. The European Working Group on Sarcopenia in Older People (EWGSOP) classifies sarcopenia into three conceptual stages based on the severity of the condition: presarcopenia is characterized by low muscle mass only without influence on muscle function. Sarcopenia is characterized by low muscle mass with low muscle function (either muscle strength or physical performance). Finally, severe sarcopenia is characterized by low muscle mass with low of the muscle function (both muscle strength or physical performance). The Asian Working Group for Sarcopenia (AWGS) also adopted this algorithm. ${ }^{6}$ In 2019, the updated EWGSOP2 classified sarcopenia into 'probable', 'confirmed', or 'severe'. EWGSOP2 elevated low muscle strength as a primary indicator of probable sarcopenia instead of low muscle mass and defined confirmed sarcopenia depending on the presence of accompanying low muscle mass. ${ }^{7)}$

Research in the economic costs associated with sarcopenia indicates that the direct expenditure was approximately $\$ 18.5$ billion 
( $\$ 10.8$ billion in men and $\$ 7.7$ billion in women) in the United States in 2000 , comprising approximately $1.5 \%$ of the country's total health expenditure. ${ }^{8)}$ The EWGSOP reported that the prevalence of sarcopenia among individuals 60-70 years of age was 5\%$13 \%$ and increased to $50 \%$ in those over 80 years of age depending on the definition and method for identifying sarcopenia., ${ }^{9,10)}$ Sarcopenia is the most significant cause of disability and frailty in the elderly, which lead to a poor quality of life. ${ }^{11)}$ In addition, sarcopenia is associated with other diseases such as diabetes, non-alcoholic fatty liver disease, and cardiovascular disease including hypertension and arterial stiffness. ${ }^{12-15)}$ Therefore, the management of sarcopenia is important for healthy aging. However, despite development efforts, to date, there remain no US Food and Drug Administration (FDA)-approved drugs for the treatment of sarcopenia (Table 1). As the population of adults aged over 60 years worldwide is predicted to expand to 2 billion by $2050,{ }^{16)}$ there is corresponding interest in the development of diagnostic tools and drugs for sarcopenia to improve the quality of life and reduce healthcare costs.

\section{MOLECULAR TARGETS FOR PHARMACOLOGICAL INTERVENTION}

The etiologies of sarcopenia are not fully understood. An imbalance between muscle protein synthesis and degradation may cause the onset of sarcopenia and various mechanisms are potentially involved in the pathogenesis of sarcopenia. Both intrinsic factors within skeletal muscle (e.g., inflammation, apoptosis, autophagy, mitochondria, neuromuscular junction, and calcium metabolism) and extrinsic factors in systemic environments (e.g., endocrine, nutritional status, and immobility $)^{10,17-21)}$ contribute to defective myogenesis, muscle atrophy, and weakness. Therefore, understanding the mechanisms in sarcopenia is essential to identify molecular targets for pharmacological treatment.

\section{MYOSTATIN}

Myostatin is the most intensively studied molecular target for mus-

Table 1. Current status of the development of drugs for sarcopenia

\begin{tabular}{|c|c|c|c|c|}
\hline Company name & Drug name & Collaborator & Target & Remark \\
\hline Novartis AG & Bimagrumab (antibody) & MorphoSys AG & $\begin{array}{l}\text { Activin receptor type } \\
2 \mathrm{~B}\end{array}$ & $\begin{array}{l}\text { Thigh muscle volume increased by week } 2 \text { and } \\
\text { was sustained throughout the treatment peri- } \\
\text { od (June 2017, phase 2). }\end{array}$ \\
\hline Regeneron Pharmaceuticals Inc. & Trevogrumab (antibody) & Sanofi S.A. & Myostatin & $\begin{array}{l}\text { Primary endpoint of phase } 2 \text { : percent change } \\
\text { in total lean body mass. }\end{array}$ \\
\hline Biophytis SAS & $\begin{array}{l}\text { Sarconeos (natural active ingredi- } \\
\text { ents) }\end{array}$ & NA & $\begin{array}{l}\text { Proto-oncogene pro- } \\
\text { tein c-MAS-1, MAS } \\
\text { receptor }\end{array}$ & $\begin{array}{l}\text { Meaningful activity in animal models of mus- } \\
\text { cular dystrophies. Good tolerability profile } \\
\text { and no serious adverse events (phase 1). }\end{array}$ \\
\hline ARMGO Pharma Inc. & ARM-210 (small molecule) & Servier & Ryanodine receptor & $\begin{array}{l}\text { Treatment of Becker and limb-girdle muscular } \\
\text { dystrophies as well as cachexia. }\end{array}$ \\
\hline Immusoft Corporation & NA (cell therapy) & $\begin{array}{l}\text { Bellicum Phar- } \\
\text { maceuticals }\end{array}$ & $\begin{array}{l}\text { Enzyme/protein re- } \\
\text { placement therapy }\end{array}$ & Immune system programming technology. \\
\hline Neurotune AG & NT-1654 (fragment of neural agrin) & NA & NA & $\begin{array}{l}\text { Low-density lipoprotein receptor-related pro- } \\
\text { tein 4, acetylcholine. NT-1654 accelerated } \\
\text { muscle reinnervation after nerve rush. }\end{array}$ \\
\hline Amgen Inc. & ATA 842 (antibody) & NA & Myostatin, activin & $\begin{array}{l}\text { ATA } 842 \text { demonstrated increased muscle mass } \\
\text { and muscle strength in the treatment of young } \\
\text { and old mice for } 4 \text { weeks. }\end{array}$ \\
\hline Vibe Pharmaceuticals LLC & VB-102 (protein) & NA & NA & $\begin{array}{l}\text { The drug can potentially regenerate muscle and } \\
\text { bones. }\end{array}$ \\
\hline MYOS RENS Technology Inc. & Peptide of follistatin & $\begin{array}{l}\text { Cloud Pharma- } \\
\text { ceuticals }\end{array}$ & $\begin{array}{l}\text { Furin, Janus kinase } 3 \text {, } \\
\text { myostatin }\end{array}$ & $\begin{array}{l}\text { Discovery of a myostatin inhibitor therapeutic } \\
\text { for the treatment of sarcopenia. }\end{array}$ \\
\hline BioViva & AAV gene therapy & NA & Myostatin & $\begin{array}{l}\text { Obtained from a natural source and has poten- } \\
\text { tial in the modulation of myostatin expression. }\end{array}$ \\
\hline Teijin Pharma Ltd. & TEI-SARM2 & NA & Androgen receptor & Selective androgen receptor modulator. \\
\hline
\end{tabular}

Source from Sarcopenia Therapeutics - Pipeline Analysis 2018 by P\&S Market Research (https://www.psmarketresearch.com/market-analysis/sarcopeniatherapeutics-pipeline-analysis).

NA, not applicable. 
cle-wasting disease. Also known as growth differentiation factor-8 (GDF-8), myostatin is a member of the transforming growth factor $\beta$ (TGF- $\beta$ ) superfamily. Myostatin is predominantly expressed in cells of skeletal muscle lineage and inhibits muscle cell growth and differentiation ${ }^{22)}$ through binding with its receptor complex activin type 2B (ACVR2B), resulting in activation of Smad signaling. ${ }^{23)}$ The loss of myostatin function induces muscle hypertrophy in children ${ }^{24)}$ and improved muscle function in animal models. ${ }^{25)}$ In addition, myostatin haploinsufficiency prevents not only aging-related declines in muscle function and but also enhances the longevity of mice. ${ }^{26)}$ Therefore, the targeting of myostatin has been proposed as a primary strategy for pharmacological interventions in muscle-wasting diseases. ${ }^{25,27)}$

The first human trial tested the myostatin inhibitor Stamulumab (MYO-029) developed by Wyeth Pharmaceuticals with Cambridge Antibody Technology Group. Stamulumab is a recombinant human antibody that neutralizes the activity of myostatin protein by preventing myostatin from binding to ACVR2B. Phase 2 clinical trials were conducted in muscular dystrophy patients but development was discontinued due to the lack of efficacy on muscle strength. Landogrozumab (LY-2495655) is a humanized monoclonal antibody developed by Eli Lilly \& Company that also neutralizes the activity of the myostatin protein. Phase 2 clinical trials have been performed in patients with sarcopenia (completed in December 2013), elective total hip replacement (completed in February 2014), and cancer cachexia (completed in January 2016), and are still under review. Increased appendicular lean body mass was reported with LY-2495655 treatment in patients aged 75 years or older who had fallen in the past year. ${ }^{28)}$ After hip fracture surgery, LY2495655 treatment induced increased appendicular lean body mass and decreased fat mass; however, the appendicular lean body mass did not reach the superiority threshold at week $12 .{ }^{29)}$

Regeneron Pharmaceuticals Inc. has developed the myostatin antibody Trevogrumab (REGN1033) in collaboration with Sanofi. Phase 2 clinical trials in sarcopenia patients were completed in February 2015 and the evaluations of its efficacy on muscle mass and function are ongoing. ${ }^{23)}$

Acceleron Pharma developed ramatercept (ACE-031) a decoy form of ACVR2B. Although the FDA awarded orphan status to this drug for muscular dystrophy and reviewed it in 2010, the development of ACE-031 was discontinued due to concerns about safety including minor nosebleeds, gum bleeding, and/or small dilated blood vessels within the skin (completed on June 2011). Alternatively, Acceleron Pharma is developing ACE-083 as a newer form of ACE-031. ACE-083 is designed for facioscapulohumeral muscular dystrophy (FSHD) and Charcot-Marie-Tooth disease (CMT) based on a modified form of human follistatin. ${ }^{23)}$ Follista- tin inhibits muscle growth signaling by binding to activins $\mathrm{A}$ and $\mathrm{B}$ and myostatin as well as other ligands in the family except for BMP9/10 ${ }^{30,31)}$ The results of phase 1 of clinical trials of ACE-083 reported in 2018 included increased muscle volume as the ACE083 dose increased. ${ }^{32)}$ Phase 2 clinical trials are ongoing to test its safety, tolerability, pharmacokinetics, and pharmacodynamics in patients with FSHD and CMT.

\section{ACTIVIN RECEPTOR}

In addition to ligands such as myostatin and activins, receptor ACVR2B has also been targeted for the development of drugs for sarcopenia. An ACVR2B antibody, which blocks the signaling pathway, was reported to induce muscle hypertrophy. ${ }^{33)}$ Novartis Institutes for BioMedical Research has developed a human monoclonal antibody, bimagrumab (BYM-338), in collaboration with MorphoSys AG. BYM-338 binds to both ACVR2A and ACVR2B and acts competitively with its ligands. In August 2013, the FDA granted breakthrough therapy designation to BYM-338 for sporadic inclusion body myositis, the most common idiopathic inflammatory myopathy that is characterized by progressive pathological muscle weakness and atrophy. BYM-338 promotes differentiation of primary human skeletal myoblasts and prevents the inhibition of differentiation induced by myostatin or activin A. BYM-338 also inhibits myostatin- or activin A-induced atrophy, thus sparing the myosin heavy chain from degradation. BYM-338 significantly increases skeletal muscle mass in mice, beyond the sole inhibition of myostatin. ${ }^{34)}$ BYM-338 is administered by intravenous infusion. Phase $2 / 3$ clinical trials of BYM-338 in patients with sporadic inclusion body myositis were completed in January 2016. However, no significant effects were observed in any objective measurements related to muscle strength or physical function. Phase 2 clinical trials of BYM-338 were also conducted in patients with hip fracture recovery or sarcopenia. In older adults with sarcopenia, BYM-338 increased muscle mass and strength and also improved mobility in those with slow walking speed. ${ }^{35)}$ Although the aforementioned study was the first to evaluate a type II activin receptor antagonist in older individuals with sarcopenia, it had some limitations. For example, the use of a gait speed cutoff of $1.0 \mathrm{~m} / \mathrm{s}$ rather than the more common $0.8 \mathrm{~m} / \mathrm{s}$ limited the number of participants in the study whose gait speed improved with bimagrumab. Moreover, the lack of introductory sessions before the gait speed or 6-minute walk tests probably led to a pervasive learning effect in the performance test results. Physical activity was not monitored, so there were also limits in understanding how exercise might interact with the drug in this population. In December 2018, Novartis discontinued development of BYM-338 for hip fracture 
recovery and sarcopenia.

Although there are currently no clear drug candidates as most of the compounds in development have had very limited efficacy in larger clinical trials, many smaller clinical trials have demonstrated that the inhibition of myostatin/ACVR2 signaling may improve muscle mass in patients with muscle wasting. As positive effects on muscle wasting through exercise training and nutritional supplementation have been reported, ${ }^{36-38)}$ hybrid therapies combining myostatin inhibitors with other approaches such as exercise and nutritional therapy could be more effective in the treatment of muscle wasting. ${ }^{23)}$

\section{EXERCISE MIMETICS}

Numerous studies have examined the positive effects of exercise on patients with sarcopenia. Exercise has consistently demonstrated improved muscle strength and function, with inconsistent effects on improving muscle mass. However, as most patients with sarcopenia have problems with physical activity, there is a limit to the ability of exercise to overcome sarcopenia. Thus, exercise mimetics (exercise pills) are a potential therapeutic strategy for sarcopenia that produce the effects of exercise without exercise. The peroxisome proliferator-activated receptor beta or delta (PPAR $\beta$ / $\delta)$ agonist GW1516 and exercise training synergistically increased oxidative myofibers and running endurance in adult mice. ${ }^{39)}$ Moreover, 4 weeks of AMP-activated protein kinase (AMPK) agonist 5-aminoimidazole-4-carboxamide ribonucleotide (AICAR) treatment alone enhanced running endurance by $44 \%$ even in sedentary mice. In addition, PPAR $\delta$ or AMPK activates robust transcription that re-programs the metabolic skeletal muscle genome. These results demonstrate that the AMPK-PPAR $\delta$ pathway can be targeted by orally active drugs to enhance training adaptation or increase endurance without exercise.

Metformin has also examined under drug repurposing for the prevention of sarcopenia with prediabetes through activation of AMPK. Metformin is commonly prescribed for the treatment of type 2 diabetes. The effects of metformin on muscle are still uncertain and its exact mechanism of action is a matter of debate. However, metformin extended the lifespan and health span with improved physical performance in model systems. ${ }^{40)}$ Thus, metformin has been studied as a potential pharmacological intervention to delay aging and the incidence of age-related diseases as well as sarcopenia.

Although most potential exercise mimetics including AMPK agonists are still in pre-clinical stages due to side effects, the development of exercise mimetics is urgently needed and should be continued for patients on bedrest or with severe sarcopenia patients with loss of physical activity.

\section{HORMONES}

Decreased circulating levels of several anabolic hormones with aging may contribute to changes in muscle mass and function in older individuals. ${ }^{41)}$ Therefore, hormonal manipulation has investigated as the basis of many of the therapies for sarcopenia. ${ }^{5)}$ In phase 2 clinical trials by GTx Inc., treatment with selective androgen receptor modulator (SARM) enobosarm (also known as ostarine, MK-2866) induced dose-dependent increases in total lean body mass with improvements in physical function in older individuals. ${ }^{42)}$ Moreover, its side effects were similar to those of the placebo, indicating that it is safer than steroids. Another phase 2 clinical trial of the SARM MK-0773 (also known as PF05314882) also increased lean body mass in women with sarcopenia, without evidence of androgenization. ${ }^{43)}$ Physical performance also tended to increase, although the difference was not statistically significant. Several patients in the treatment group experienced elevated transaminase levels that resolved after discontinuing the study. Despite growing evidence linking age-related hormonal changes to the development of sarcopenia, it is still too early to determine the clinical efficacy of hormonal supplementation for the management of sarcopenia. ${ }^{44)}$

\section{NATURAL COMPOUNDS}

Since sarcopenia is an age-related disease, natural compounds with anti-aging effects have been assessed for anti-sarcopenic properties. Ursolic acid, a pentacyclic triterpenoid enriched in apples, reduced muscle atrophy and stimulated muscle hypertrophy in mice by enhancing skeletal muscle insulin/IGF-1 signaling and inhibiting atrophy-associated mRNA expression. ${ }^{45)}$ Furthermore, the effects were accompanied by reductions in adiposity and levels of fasting blood glucose, and plasma cholesterol and triglycerides.

Tomatidine improves muscular strength and decreases adiposity. ${ }^{46)}$ Tomatidine, abundant in unripe green tomatoes, is a metabolite of $\alpha$-tomatine. Supplementation with tomatidine in old mice significantly reduced age-dependent declines in skeletal muscle mass, strength, and quality. ${ }^{47)}$

Both ursolic acid and tomatidine generate hundreds of small positive and negative changes in mRNA levels in aged skeletal muscle, with remarkably similar mRNA expression signatures. ${ }^{47)}$ Ursolic acid and tomatidine in aged skeletal muscle reportedly repressed a subset of the mRNAs positively regulated by activating transcription factor 4 (ATF4), a basic leucine zipper (bZIP) transcription factor subunit regulating oxidative and other stress responses. $^{48)}$ 
High-energy skeletal muscle tissue relies upon mitochondria for energy production and contractile function; however, mitochondrial function declines with aging. ${ }^{49)}$ The association between sarcopenia and mitochondria may also inform new and effective treatments for sarcopenia. ${ }^{50)}$ Mitophagy plays a significant role in mitochondrial quality control in muscles; therefore, mitophagy-inducing agents may have anti-sarcopenic functions. ${ }^{51)}$ Urolithin A is a metabolically transformed compound from a group of natural compounds, ellagitannins (ETs), which are found in pomegranates, as well as nuts and berries. ${ }^{52)}$ Urolithin A has been shown to induce mitophagy and prolong lifespan in $C$. elegans and increase muscle function in rodents. ${ }^{52)}$

The results of studies in model animals suggest that supplementation with these natural compounds may ameliorate sarcopenia in older individuals, especially when clinically assessed with other medical treatment options.

\section{CONCLUSION}

According to reports from the FDA in 2017, ${ }^{53)}$ many patients with sarcopenia are uncertain if their symptoms worsen due to sarcopenia or as a natural result of aging. Therefore, older individuals should recognize sarcopenia as a disease to prevent or treat. As a new disease code for sarcopenia was established in ICD-10-CM (M62.84) in October 2016, other countries will also soon accept sarcopenia as a disease entity.

As a result of sarcopenia, patients have difficulty in performing basic physical tasks such as standing and walking long distances. Moreover, balance problems, falling, fatigue, and muscle pain, along with comorbid conditions such as arthritis, make them inactive. In addition to physical limitations, patients with sarcopenia experience emotional impacts such as fear of injury and embarrassment of their physical limitations, limited social interactions and feelings of isolation, and difficulties caring for themselves and living independently. ${ }^{53)}$ Therefore, the development of preventive and therapeutic strategies against sarcopenia is imperative for healthy aging. Future large-scale clinical trials are essential to develop precision medicine reflecting individual patient characteristics since the cause and clinical features vary between patients. Comprehensive strategies together with pharmacological and non-pharmacological intervention including exercise, physical therapy, dietetic regulation, lifestyle modification, and emotional support may be more effective against sarcopenia. ${ }^{53)}$

\section{CONFLICT OF INTEREST DISCLOSURES}

The researchers claim no conflicts of interest.

\section{REFERENCES}

1. Rosenberg IH. Sarcopenia: origins and clinical relevance. J Nutr 1997;127(5 Suppl):990S-991S.

2 .McGregor RA, Cameron-Smith D, Poppitt SD. It is not just muscle mass: a review of muscle quality, composition and metabolism during ageing as determinants of muscle function and mobility in later life. Longev Healthspan 2014;3:9.

3. Lang T, Streeper T, Cawthon P, Baldwin K, Taaffe DR, Harris TB. Sarcopenia: etiology, clinical consequences, intervention, and assessment. Osteoporos Int 2010;21:543-59.

4. Cao L, Morley JE. Sarcopenia is recognized as an independent condition by an International Classification of Disease, Tenth Revision, Clinical Modification (ICD-10-CM) code. J Am Med Dir Assoc 2016;17:675-7.

5. Dennison EM, Sayer AA, Cooper C. Epidemiology of sarcopenia and insight into possible therapeutic targets. Nat Rev Rheumatol 2017;13:340-7.

6. Chen LK, Liu LK, Woo J, Assantachai P, Auyeung TW, Bahyah KS, et al. Sarcopenia in Asia: consensus report of the Asian Working Group for Sarcopenia. J Am Med Dir Assoc 2014; 15:95-101.

7. Cruz-Jentoft AJ, Bahat G, Bauer J, Boirie Y, Bruyere O, Cederholm T, et al. Sarcopenia: revised European consensus on definition and diagnosis. Age Ageing 2019;48:16-31.

8. Janssen I, Shepard DS, Katzmarzyk PT, Roubenoff R. The healthcare costs of sarcopenia in the United States. J Am Geriatr Soc 2004;52:80-5.

9. Cruz-Jentoft AJ, Baeyens JP, Bauer JM, Boirie Y, Cederholm T, Landi F, et al. Sarcopenia: European consensus on definition and diagnosis: report of the European Working Group on Sarcopenia in older people. Age Ageing 2010;39:412-23.

10. Cruz-Jentoft AJ, Landi F, Schneider SM, Zuniga C, Arai H, Boirie $\mathrm{Y}$, et al. Prevalence of and interventions for sarcopenia in ageing adults: a systematic review. Report of the International Sarcopenia Initiative (EWGSOP and IWGS). Age Ageing 2014; 43:748-59.

11. Roubenoff R. Sarcopenia: a major modifiable cause of frailty in the elderly. J Nutr Health Aging 2000;4:140-2.

12. Morley JE, Malmstrom TK, Rodriguez-Manas L, Sinclair AJ. Frailty, sarcopenia and diabetes. J Am Med Dir Assoc 2014;15: 853-9.

13. Hong HC, Hwang SY, Choi HY, Yoo HJ, Seo JA, Kim SG, et al. Relationship between sarcopenia and nonalcoholic fatty liver disease: the Korean Sarcopenic Obesity Study. Hepatology 2014;59:1772-8.

14. Han K, Park YM, Kwon HS, Ko SH, Lee SH, Yim HW, et al. Sar- 
copenia as a determinant of blood pressure in older Koreans: findings from the Korea National Health and Nutrition Examination Surveys (KNHANES) 2008-2010. PLoS One 2014;9: e86902.

15. Snijder MB, Henry RM, Visser M, Dekker JM, Seidell JC, Ferreira I, et al. Regional body composition as a determinant of arterial stiffness in the elderly: the Hoorn Study. J Hypertens 2004;22: 2339-47.

16. United Nations. World population ageing 2013. New York: United Nations; 2013.

17. Kalinkovich A, Livshits G. Sarcopenia: the search for emerging biomarkers. Ageing Res Rev 2015;22:58-71.

18. Ilich JZ, Kelly OJ, Inglis JE, Panton LB, Duque G, Ormsbee MJ. Interrelationship among muscle, fat, and bone: connecting the dots on cellular, hormonal, and whole body levels. Ageing Res Rev 2014;15:51-60.

19. Kob R, Bollheimer LC, Bertsch T, Fellner C, Djukic M, Sieber CC, et al. Sarcopenic obesity: molecular clues to a better understanding of its pathogenesis? Biogerontology 2015;16:15-29.

20. Marzetti E, Calvani R, Lorenzi M, Marini F, D’Angelo E, Martone AM, et al. Serum levels of C-terminal agrin fragment (CAF) are associated with sarcopenia in older hip fractured patients. Exp Gerontol 2014;60:79-82.

21. Sakuma K, Aoi W, Yamaguchi A. Current understanding of sarcopenia: possible candidates modulating muscle mass. Pflugers Arch 2015;467:213-29.

22. McPherron AC, Lee SJ. Double muscling in cattle due to mutations in the myostatin gene. Proc Natl Acad Sci U S A 1997;94: 12457-61.

23. Saitoh M, Ishida J, Ebner N, Anker SD, Springer J, Haehling SV. Myostatin inhibitors as pharmacological treatment for muscle wasting and muscular dystrophy. J Cachexia Sarcopenia Muscle Clin Rep 2017;2:e00037.

24. Schuelke M, Wagner KR, Stolz LE, Hubner C, Riebel T, Komen W, et al. Myostatin mutation associated with gross muscle hypertrophy in a child. N Engl J Med 2004;350:2682-8.

25. Bogdanovich S, Krag TO, Barton ER, Morris LD, Whittemore LA, Ahima RS, et al. Functional improvement of dystrophic muscle by myostatin blockade. Nature 2002;420:418-21.

26. Mendias CL, Bakhurin KI, Gumucio JP, Shallal-Ayzin MV, Davis CS, Faulkner JA. Haploinsufficiency of myostatin protects against aging-related declines in muscle function and enhances the longevity of mice. Aging Cell 2015;14:704-6.

27. Wagner KR, McPherron AC, Winik N, Lee SJ. Loss of myostatin attenuates severity of muscular dystrophy in mdx mice. Ann Neurol 2002;52:832-6.

28. Becker C, Lord SR, Studenski SA, Warden SJ, Fielding RA, Rec- knor CP, et al. Myostatin antibody (LY2495655) in older weak fallers: a proof-of-concept, randomised, phase 2 trial. Lancet Diabetes Endocrinol 2015;3:948-57.

29. Woodhouse L, Gandhi R, Warden SJ, Poiraudeau S, Myers SL, Benson CT, et al. A phase 2 randomized study investigating the efficacy and safety of myostatin antibody LY2495655 versus placebo in patients undergoing elective total hip arthroplasty. J Frailty Aging 2016;5:62-70.

30. Townson SA, Martinez-Hackert E, Greppi C, Lowden P, Sako D, Liu J, et al. Specificity and structure of a high affinity activin receptor-like kinase 1 (ALK1) signaling complex. J Biol Chem 2012;287:27313-25.

31. Pearsall R, Widrick J, Cotton E, Sako D, Liu J, Davies M, et al. ACE-083 increases muscle hypertrophy and strength in C57BL/6 mice. Neuromuscul Disord 2015;25(Supp1 2):S218.

32. Glasser CE, Gartner MR, Wilson D, Miller B, Sherman ML, Attie KM. Locally acting ACE-083 increases muscle volume in healthy volunteers. Muscle Nerve 2018;57:921-6.

33. Lee SJ, Huynh TV, Lee YS, Sebald SM, Wilcox-Adelman SA, Iwamori N, et al. Role of satellite cells versus myofibers in muscle hypertrophy induced by inhibition of the myostatin/activin signaling pathway. Proc Natl Acad Sci U S A 2012;109:E2353-60.

34. Lach-TrifilieffE, Minetti GC, Sheppard K, Ibebunjo C, Feige JN, Hartmann S, et al. An antibody blocking activin type II receptors induces strong skeletal muscle hypertrophy and protects from atrophy. Mol Cell Biol 2014;34:606-18.

35. Rooks D, Praestgaard J, Hariry S, Laurent D, Petricoul O, Perry RG, et al. Treatment of sarcopenia with bimagrumab: results from a phase II, randomized, controlled, proof-of-concept study. J Am Geriatr Soc 2017;65:1988-95.

36. Argiles JM, Busquets S, Lopez-Soriano FJ, Costelli P, Penna F. Are there any benefits of exercise training in cancer cachexia? J Cachexia Sarcopenia Muscle 2012;3:73-6.

37. Coats AJ. Research on cachexia, sarcopenia and skeletal muscle in cardiology.J Cachexia Sarcopenia Muscle 2012;3:219-23.

38. Gould DW, Lahart I, Carmichael AR, Koutedakis Y, Metsios GS. Cancer cachexia prevention via physical exercise: molecular mechanisms. J Cachexia Sarcopenia Muscle 2013;4:111-24.

39. Narkar VA, Downes M, Yu RT, Embler E, Wang YX, Banayo E, et al. AMPK and PPARdelta agonists are exercise mimetics. Cell 2008;134:405-15.

40. Kulkarni AS, Brutsaert EF, Anghel V, Zhang K, Bloomgarden N, Pollak M, et al. Metformin regulates metabolic and nonmetabolic pathways in skeletal muscle and subcutaneous adipose tissues of older adults. Aging Cell 2018;17:e12723.

41. Tenover JS. Effects of testosterone supplementation in the aging male. J Clin Endocrinol Metab 1992;75:1092-8. 
42. Dalton JT, Barnette KG, Bohl CE, Hancock ML, Rodriguez D, Dodson ST, et al. The selective androgen receptor modulator GTx-024 (enobosarm) improves lean body mass and physical function in healthy elderly men and postmenopausal women: results of a double-blind, placebo-controlled phase II trial. J Cachexia Sarcopenia Muscle 2011;2:153-61.

43. Papanicolaou DA, Ather SN, Zhu H, Zhou Y, Lutkiewicz J, Scott $\mathrm{BB}$, et al. A phase IIA randomized, placebo-controlled clinical trial to study the efficacy and safety of the selective androgen receptor modulator (SARM), MK-0773 in female participants with sarcopenia. J Nutr Health Aging 2013;17:533-43.

44. Kamel HK, Maas D, Duthie EH Jr. Role of hormones in the pathogenesis and management of sarcopenia. Drugs Aging 2002;19:865-77.

45. Kunkel SD, Suneja M, Ebert SM, Bongers KS, Fox DK, Malmberg SE, et al. mRNA expression signatures of human skeletal muscle atrophy identify a natural compound that increases muscle mass. Cell Metab 2011;13:627-38.

46. Dyle MC, Ebert SM, Cook DP, Kunkel SD, Fox DK, Bongers $\mathrm{KS}$, et al. Systems-based discovery of tomatidine as a natural small molecule inhibitor of skeletal muscle atrophy. J Biol Chem 2014;289:14913-24.

47. Ebert SM, Dyle MC, Bullard SA, Dierdorff JM, Murry DJ, Fox DK, et al. Identification and small molecule inhibition of an activating transcription factor 4 (ATF4)-dependent Pathway to age-related skeletal muscle weakness and atrophy. J Biol Chem 2015;290:25497-511.

48. Rutkowski DT, Kaufman RJ. All roads lead to ATF4. Dev Cell 2003;4:442-4.

49. Short KR, Bigelow ML, Kahl J, Singh R, Coenen-Schimke J, Raghavakaimal S, et al. Decline in skeletal muscle mitochondrial function with aging in humans. Proc Natl Acad Sci U S A 2005; 102:5618-23.

50. Waltz TB, Fivenson EM, Morevati M, Li C, Becker KG, Bohr VA, et al. Sarcopenia, aging and prospective interventional strategies. Curr Med Chem 2018;25:5588-96.

51. Romanello V, Sandri M. Mitochondrial quality control and muscle mass maintenance. Front Physiol 2016;6:422.

52. Ryu D, Mouchiroud L, Andreux PA, Katsyuba E, Moullan N, Nicolet-Dit-Felix AA, et al. Urolithin A induces mitophagy and prolongs lifespan in C. elegans and increases muscle function in rodents. Nat Med 2016;22:879-88.

53. US Food and Drug Administration. The voice of the patient: sarcopenia: a series of reports from FDA's patient-focused drug development initiative [Internet]. Silver Spring: US Food and Drug Administration; 2017 [cited 2019 Aug 30]. Available from: https:// www.fda.gov/industry/prescription-drug-user-fee-amendments/ voice-patient-series-reports-fdas-patient-focused-drug-development-initiative. 